\title{
A Computational Insight on Designing Low Electronic Energy Gap (Benzothiadiazole/ Benzoselenadiazole - Pyrrole) copolymers
}

\author{
Pinar Seyiddanlioglu Sirin ${ }^{1}$, Pervin $\mathrm{Civcir}^{2}$, and canan unaleroglu ${ }^{1}$ \\ ${ }^{1}$ Hacettepe Universitesi \\ ${ }^{2}$ Ankara University
}

September 2, 2020

\begin{abstract}
Organic conductive polymers have great significance due to their wide range of applications in optoelectronics and material sciences. In this study, pyrrole-benzothiadiazole/benzoselenadiazole based type green polymers were undertaken computational work to investigate the solubility of polymers. Structural, electronic, and optical properties of eight different polymers were predicted using DFT and TD-DFT at B3LYP/6-31G level on semi-empirical PM6-optimized geometries. It has been shown that the calculation results of synthesized green polymers are in great agreement with the experimental results. Alkylated 4,7-di(1H-pyrrol-2-yl)benzo-[c][1,2,5]thiadiazole (PB1) and 4,7-di(1H-pyrrol-2-yl)benzo[c][1,2,5]selenadiazole (PB7) monomers were studied to investigate the effect of alkyl chains on their electronic and optical properties. Butyl substituted more soluble polymers were shown to have low electronic energy gaps (1.27-1.55 eV). Moreover, the electronic energy gap values of the studied polymeric structures are in the appropriate range of technological applications (1.24-2.18 eV). The approach utilized in this study can be used to design new semi-conducting polymers.
\end{abstract}

\section{Hosted file}

Manuscript.docx available at https://authorea.com/users/355734/articles/478870-acomputational-insight-on-designing-low-electronic-energy-gap-benzothiadiazolebenzoselenadiazole-pyrrole-copolymers 